\title{
AIM2(-1)/HT001(-1)/TAF1B(-1) Frameshift Peptide Vaccine
}

National Cancer Institute

\section{Source}

National Cancer Institute. AIM2(-1)/HT001(-1)/TAF1B(-1) Frameshift Peptide Vaccine.

NCI Thesaurus. Code C99129.

A cancer vaccine containing the three frame shift peptides (FSP) AIM2(-1), HT001(-1) and

TAF1B(-1), with potential immunomodulating activity. Upon administration, the AIM2(-

1)/HT001(-1)/TAF1B(-1) FSP vaccine may induce an immune response against

microsatellite instability (MSI) colorectal cancer-associated antigens. Frame shift mutations of AIM2 (absent in melanoma 2, an interferon-inducible protein), HT001 (asteroid homolog 1 or ASTE1, with an unknown function) and TAF1B (TATA box-binding protein-associated RNA polymerase I B, a transcription factor) are seen in MSI-positive colorectal cancers and may be associated with malignant transformation, tumor progression and the presence of tumor-infiltrating lymphocytes. These FSPs all have one-base deletions. 